\title{
La lèpre n'est pas une maladie du passé
}

> Cette revue synthétique présente, à partir des données épidémiologiques et de recherche les plus récentes sur le sujet, les enjeux actuels de la lutte contre la lèpre. A côté des progrès importants réalisés dans l'étude génomique du bacille de la lèpre, ainsi que dans la compréhension des mécanismes fondamentaux de sensibilité individuelle à la maladie, la stagnation de l'incidence des nouveaux cas annuels observée depuis une dizaine d'années est source d'interrogations. Si l'efficacité des traitements recommandés est confirmée par un faible taux de rechute, le tarissement espéré des nouveaux cas par réduction de la contagiosité des cas traités tarde à se manifester. Des études récentes sont en faveur de l'efficacité d'une prophylaxie par prise unique de rifampicine et/ ou vaccination par le BCG des sujets contacts, ce qui pourrait permettre une accélération de la réduction de l'incidence de la maladie. Toutefois, aucune prise de position officielle sur ces travaux ne valide la mise en œuvre de telles stratégies. Du fait d'une ambiguïté dans l'expression des objectifs opérationnels de prise en charge de la lèpre, "l'élimination en tant que problème de santé publique » de la maladie ne devant notamment pas être confondue avec une « éradication » qui n'est nullement d'actualité, une démobilisation dans la lutte contre la lèpre est à redouter. <

La lèpre, ou maladie de Hansen, est une maladie qui semble ne plus guère faire parler d'elle aujourd'hui. Le nombre de publications scientifiques s'y intéressant se réduit d'année en année. Cela a d'ailleurs même pu aboutir à la disparition pure et simple de revues médicales qui lui étaient autrefois dédiées (disparition notamment en 2005 , de la revue « phare » qu'était l'International Journal of Leprosy). On peut estimer que cet état de chose est

\section{Antoine Mahé}

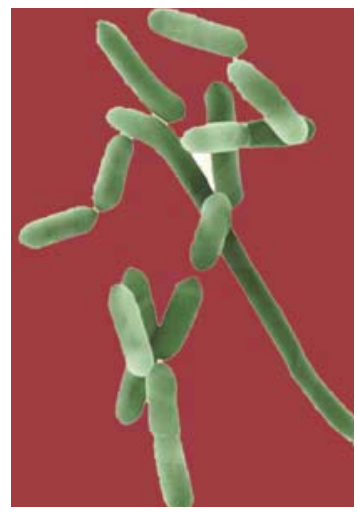

Service de dermatologie, Centre hospitalier Louis Pasteur, 39 avenue de la Liberté, 68024 Colmar, France. antoine.mahe@ch-colmar.fr

le juste reflet des importants - et réels - succès obtenus dans la lutte organisée contre ce fléau, qui n'est indiscutablement plus ce qu'il a pu être. Mais, pour une maladie dont aucune donnée n'indique qu'elle est éradiquée, ou seulement en passe de l'être, il paraît licite de faire le point sur l'état des connaissances la concernant aujourd'hui, ne seraitce qu'au regard des plus de 200000 nouveaux cas encore répertoriés de par le monde chaque année [1]. Nous souhaitons présenter ici un panorama des principales avancées récentes ainsi que des enjeux concernant la lutte contre la lèpre aujourd'hui.

\section{Données épidémiologiques}

L'introduction au début des années 1980 de protocoles thérapeutiques « courts » (de 6 mois à 2 ans selon la forme clinique) comportant de la rifampicine et permettant de guérir bactériologiquement les malades avec un taux de rechute extrêmement faible, alors qu'il s'agissait volontiers autrefois de traitements «à vie », a bouleversé l'épidémiologie mondiale de la maladie [2]. Dès lors qu'on passait, pour évaluer le nombre de malades, de files actives cumulatives à un reflet de l'incidence annuelle de nouveaux cas, le nombre de «malades de la lèpre » (c'est-à-dire, en pratique, de malades diagnostiqués et traités par antibacillaires) a considérablement chuté, passant de plus cinq millions en 1985 à moins de trois cent mille cas annuels depuis 2005 (Tableau I).

Un espoir implicite de cette stratégie était que, puisque le risque d'exposition des sujets sains à des malades actifs disséminateurs de bacilles diminuait de façon drastique, l'incidence des nouveaux cas devait se tarir en quelque sorte d'elle-même du fait d'une réduction « mathématique » du risque de contagion. En fait, la réalité est qu'on est confronté, depuis environ une décennie, à une stagnation de l'incidence recensée de la maladie (215 656 nouveaux cas en 2013), qui 


\begin{tabular}{|c|c|c|c|c|c|c|c|c|}
\hline $\begin{array}{l}\text { Régions } \\
\text { de l'OMS }\end{array}$ & 2006 & 2007 & 2008 & 2009 & 2010 & 2011 & 2012 & 2013 \\
\hline Afrique & 34480 & 34468 & 29814 & 28935 & 25345 & 20213 & 20599 & 20911 \\
\hline Amériques & 47612 & 42135 & 41891 & 40474 & 37740 & 36832 & 36178 & 33084 \\
\hline Asie du Sud-Est & 174118 & 171576 & 167505 & 166115 & 156254 & 160132 & 166445 & 155385 \\
\hline Méditerranée orientale & 3261 & 4091 & 3938 & 4029 & 4080 & 4357 & 4235 & 1680 \\
\hline Pacifique occidental & 6190 & 5863 & 5859 & 5243 & 5055 & 5092 & 5400 & 4596 \\
\hline Total & 265661 & 258133 & 249007 & 244796 & 228474 & 226626 & 232857 & 215656 \\
\hline
\end{tabular}

Tableau I. Nombre de nouveaux cas de lèpre dépistés dans le monde : tendances observées par régions de l’0MS, de 2006 à 2013 [2]. ${ }^{1}$ Aucun rapport pour la région européenne.

n'est d'ailleurs elle-même sans doute pas tout à fait assimilable à son incidence réelle. À ce rythme, des modélisations ont montré qu'une élimination complète de la maladie ne serait pas à espérer avant au moins plusieurs décennies [3]. Nous verrons plus loin quelles solutions pourraient être envisagées pour contrer ce phénomène qui a pu contrarier certains optimismes initiaux excessifs.

Sur un autre plan, il convient de relever que ces statistiques flatteuses ne comptabilisent que les malades actifs traités par antibiotiques, autrement dit pas les patients atteints d'invalidités liées à la lèpre, pourtant nombreux. Elles concernaient en 2013 environ 13000 patients sur les 215000 nouveaux cas diagnostiqués, pour un total cumulé de personnes handicapées par la lèpre très grossièrement estimé à deux à trois millions d'individus. La proportion de nouveaux patients atteints d'invalidité de stade $2^{1}$ est, de plus, remarquablement stable depuis une dizaine d'années. En pratique, la prise en charge de ces patients porteurs d'invalidités relève pour beaucoup d'initiatives issues du milieu caritatif.

\section{Des progrès majeurs dans la compréhension de la structure génomique de Mycobacterium leprae}

Le bacille responsable de la lèpre, Mycobacterium leprae, présente certaines particularités étonnantes. Son génome, totalement séquencé en 2000 , se distingue par la présence particulièrement abondante de pseudogènes dénués de toute capacité de codage efficace (précisément, 1133 pseudogènes pour 1614 gènes codants) [4]. Cela n'est sans doute pas étranger à certaines originalités remarquables du comportement de $M$. leprae comme son temps de doublement élevé (environ 12 jours, contre par exemple 20 heures pour M. tuberculosis), phénomène lui-même probablement à l'origine des délais extrêmes susceptibles de jalonner l'histoire naturelle de la maladie, comme la durée d'incubation qui peut atteindre vingt ans, ou encore les délais de rechute après traitement qui peuvent, quant à eux, dépasser dix ans.

\footnotetext{
${ }^{1}$ Patients présentant des séquelles neurologiques visibles.
}

Rappelons par ailleurs que M. leprae n'est toujours pas cultivable in vitro. Une analyse par PCR spécifique est certes disponible, mais reste de faible intérêt diagnostique. Cette technique apparaît surtout utile pour dépister chez les malades multibacillaires une résistance à l'un ou l'autre des principaux antibiotiques utilisables dans son traitement [5].

Le monomorphisme génomique de $M$. leprae apparaît de plus tout à fait particulier, avec une diversité génétique des souches à l'échelon mondial réduite à quelques dizaines de polymorphismes nucléotidiques [6]. On en a ainsi déduit que l'ensemble des cas mondiaux étaient issus d'un clone unique, dont la dissémination s'est effectuée à partir d'une souche originelle issue probablement de Afrique de l'Est, avec contamination progressive des autres régions en fonction des migrations humaines. Ces souches disséminées conservent une similitude génétique supérieure à 99,99\% [7].

Les études génomiques ont par ailleurs permis de documenter le rôle de réservoirs animaux potentiels. Ainsi, dans le sud des États-Unis, en Louisiane, où le tatou à neuf bandes (armadillo) pullule et est susceptible d'être infecté par une souche de $M$. leprae ayant un génotype conforme à celui de cette zone géographique, il a été rapporté une contamination humaine s'exprimant par une lèpre/maladie ne pouvant être distinguée des formes non zoonotiques [8].

Pour finir, un variant de M. leprae (M. lepromatosis, présentant une variation génomique d'environ $9 \%$ par rapport à $M$. leprae) a été récemment décrit au Mexique, et est à l'origine d'une forme clinique particulière cantonnée à cette zone géographique : la lèpre lépromateuse diffuse dite « de Lucio ». Cette pathologie est caractérisée notamment par une invasion massive des cellules endothéliales des petits vaisseaux par la mycobactérie. Elle est susceptible 


\begin{tabular}{|c|c|c|c|c|}
\hline \multirow{2}{*}{$\begin{array}{l}\text { Médica- } \\
\text { ments }\end{array}$} & \multirow{2}{*}{$\begin{array}{l}\text { Dose } \\
\text { par } \\
\text { prise }\end{array}$} & \multirow[b]{2}{*}{ Rythme* } & \multicolumn{2}{|c|}{$\begin{array}{c}\text { Durée du traitement } \\
\text { (en mois) }\end{array}$} \\
\hline & & & $\begin{array}{l}\text { Lèpre } \\
\text { pauci- } \\
\text { bacillaire }\end{array}$ & $\begin{array}{l}\text { Lèpre } \\
\text { multi- } \\
\text { bacillaire }\end{array}$ \\
\hline Rifampicine & $600 \mathrm{mg}$ & Mensuel & 6 & 12 \\
\hline Dapsone & $100 \mathrm{mg}$ & Journalier & 6 & 12 \\
\hline Clofazimine & $\begin{array}{l}300 \mathrm{mg} \\
+50 \mathrm{mg}\end{array}$ & $\begin{array}{l}\text { Mensuel } \\
\text { Journalier }\end{array}$ & 0 & 12 \\
\hline
\end{tabular}

Tableau II. Protocoles de polychimiothérapie de la lèpre recommandés par l'OMS.

* Dans les pays développés, la rifampicine est généralement prise quotidiennement.

de se compliquer de manifestations vasculaires cliniques [9]. Ce variant génomique dériverait d'un ancêtre commun avec M. leprae, avec lequel il partage heureusement une sensibilité commune aux antibiotiques antilépreux [10].

\section{Physiopathologie de la maladie lépreuse : un va-et-vient entre génétique et immunologie individuelles}

On comprend mieux aujourd'hui les mécanismes fondamentaux conduisant certains sujets exposés au bacille de Hansen à être malades, ou non $[11,12]$. Une prédisposition génétique est un paramètre fondamental de survenue de la maladie ainsi que de ses modalités d'expression. Globalement, en cas d'exposition à M. leprae, seule une minorité des individus, estimée à $5 \%$, sont susceptibles de développer une lèpre/maladie. La réponse immune se fait schématiquement à deux niveaux distincts. Lors du contact de l'organisme avec $M$. leprae, une immunité innée à médiation monocytaire (sous dépendance notamment des gènes PARKIN et $N R A M P 1^{2}$ ) [19] permet chez la plupart des sujets l'élimination définitive du germe. Parmi les sujets infestés à un niveau infraclinique, la réponse immune innée est encore susceptible de permettre un contrôle complet de l'infection. Chez la fraction résiduelle de sujets n'ayant pu éradiquer le germe, une réponse immune à médiation cellulaire, cette fois dépendante des lymphocytes $T$, entre en jeu (immunité adaptative), également sous dépendance génétique, mais à partir d'autres locus (HLA-DR2 et 3 , région chromosomique $10 \mathrm{pl} 3$ notamment). En cas de réponse lymphocytaire T importante vis-à-vis de M. leprae se développe alors une lèpre de forme tuberculoïde/paucibacillaire. En revanche, dans le cas d'une déficience de l'immunité lymphocytaire T, une forme lépromateuse/multibacillaire se déclare.

\footnotetext{
${ }^{2}$ PARK2, gène identifié comme responsable de certaines formes de la maladie de Parkinson, code pour une enzyme, la parkine, qui participe à l'une des voies majeures de dégradation des protéines cellulaires, la cascade d'ubiquitination. Le gène NRAMPI (natural resistance associated macrophage protein 1) code pour une protéine intégrale des cellules phagocytaires.
}

La polychimiothérapie de la lèpre : une efficacité élevée, un taux de rechute faible et une prévention efficace des résistances acquises

Les principaux antibiotiques utilisés dans le traitement de la lèpre sont la dapsone (ou disulone), antibiotique de la classe des sulfones doté d'une activité bactériostatique sur $M$. leprae et connu depuis le début des années 1950 , et surtout la rifampicine, d'efficacité bactéricide majeure puisqu'une seule dose de ce composé tue plus de $99,9 \%$ des bacilles hébergés par un sujet multibacillaire (ce qui suffit par la même occasion à rendre le malade non contagieux). La clofazimine est un autre composé bactériostatique utilisé chez les patients multibacillaires ou en cas d'intolérance à la dapsone chez les paucibacillaires.

Les principes bactériologiques du traitement antibiotique de la lèpre sont calqués sur ceux de la tuberculose. On admet qu'il existe, pour toute souche, y compris sauvage, de M. leprae, une fraction de bacilles qui résistent à l'un ou l'autre des antibiotiques disponibles. Le risque de cumulation d'une résistance à plusieurs composés apparaît insignifiant. L'utilisation simultanée de plusieurs antibiotiques, outre qu'elle est susceptible d'accélérer la guérison, a pour but principal d'éviter la sélection de souches résistantes à l'un et/ou l'autre d'entre eux (Tableau II).

La bonne nouvelle est que toutes les données disponibles sur la sensibilité des souches bactériennes de la petite proportion de patients rechutant après traitement confirment l'excellent effet préventif d'une polychimiothérapie menée selon ces modèles sur la survenue de résistances acquises. Ceci est particulièrement bienvenu dans la mesure où le nombre d'antibiotiques ayant montré une efficacité dans la lèpre reste restreint (en dehors de ceux déjà évoqués, on peut également citer l'ofloxacine, la minocycline, la clarithromycine, et plus récemment la rifapentine), et qu'en outre aucun des protocoles de substitution utilisant l'un ou l'autre de ces «nouveaux » composés n'a montré un taux de succès comparable à ceux prônés.

\section{Un domaine qui évolue peu : celui de la prise en charge clinique des patients}

En fait, si les données brutes d'incidence et de prise en charge sont globalement satisfaisantes, d'importants problèmes persistent. Passons rapidement - mais ne l'omettons tout de même pas - sur le risque de diagnostic en excès de la lèpre : on sait par exemple que, devant une tache claire de la peau (maître signe de la lèpre sur une peau fortement 


\section{Rappel sur l'expression clinique de la maladie}

Schématiquement, la lèpre peut être vue comme une maladie neurologique dont la présentation initiale est généralement dermatologique. Les signes cutanés susceptibles de permettre un diagnostic précoce de la maladie précèdent ainsi la plupart du temps l'atteinte des gros nerfs périphériques, dont la destruction est susceptible d'entraîner des lésions graves (paralysies, anesthésies), elles-mêmes à l'origine des invalidités et mutilations qui font toute la gravité de la maladie.

Sur le plan clinique, la lèpre est une maladie dont l'expression varie grandement selon la réponse immune du sujet atteint. Selon la qualité de cette réponse, on distingue les formes tuberculoïdes/paucibacillaires, avec une immunité $T$ relativement efficace, et les formes lépromateuses/multibacillaires que caractérise une immunité vis-à-vis de M. Leprae faible voire absente. Une autre particularité remarquable de la maladie réside dans l'instabilité immunologique de nombreux patients, dont beaucoup sont en réalité situés sur le plan immun entre ces deux pôles (malades dits «borderline »), avec le risque chez ces patients de survenue de regains d'immunité, sources de réactions cliniques violentes (dites « de réversion ») qui peuvent être extrêmement dommageables pour les nerfs.

pigmentée), la chance statistique d'être en présence d'une lèpre est au minimum quatre cent fois plus faible que celle d'être en présence d'une dermatose hypochromiante banale, et ce même en zone d'endémie [13]. II nous apparaît peu douteux que parmi les cas de «lèpre monolésionnelle » susceptibles de bénéficier selon l'Organisation mondiale de la santé (OMS) d'un traitement minute, une proportion notable relève en fait d'un diagnostic erroné. On peut cependant admettre que, dans une certaine mesure, le risque d'un traitement injustifié (qui reste faible) outrepasse celui d'une méconnaissance de cas authentiques. L'insuffisance de formation des agents de santé périphériques à une dermatologie élémentaire, agents qui sont aujourd'hui dans de nombreux pays censés gérer cette maladie, ne saurait toutefois être méconnue. C'est particulièrement vrai si l'on espère faire un diagnostic précoce de la maladie avant l'installation de troubles neurologiques, et donc devant des signes qui restent purement cutanés.

Mais c'est surtout à propos des réactions lépreuses et de leurs conséquences que le problème n'est pas loin de rester entier. En effet, il apparaît particulièrement regrettable de «guérir 》 des malades sur un plan bactériologique, tout en étant susceptible de les aggraver par le biais de réactions assez imprévisibles, lesquelles, si elles ne sont pas prises en charge d'une façon spécifique urgente et énergique, peuvent être source de graves infirmités - et parfois même malgré ces interventions (voir Encadré). Le dépistage précoce de réactions latentes, qui, malgré un caractère peu bruyant, n'en sont peut-être pas moins délétères, reste un enjeu qui, à l'heure actuelle, n'a pas reçu de réponse satisfaisante. Le projet TENLEP (treatment of early neuropathy in leprosy) actuellement en cours changera peut-être la donne concernant ce sujet difficile [14].
D’une façon générale, on peut également regretter le peu de transfert des progrès réalisés dans d'autres domaines de la médecine vers le champ parfois quelque peu autarcique de la léprologie, tels que la prise en charge de la douleur, ou encore l'apport potentiel des techniques modernes d'imagerie des nerfs que sont l'échographie ou l'IRM.

\section{Limites des stratégies de l'Organisation mondiale de la santé}

Au début des années 1990, l'Organisation mondiale de la santé, qui représente la référence officielle et incontournable dans la prise en charge de la lèpre, a clairement centré sa stratégie sur un objectif de réduction de la prévalence de la maladie. L'objectif annoncé en 1991 était donc "d'éliminer la lèpre en tant que problème de santé publique », ce qui en pratique correspondait à l'obtention d'une prévalence inférieure à $1 / 10000$ cas en population générale. Ce taux était rendu envisageable, et en fin de compte réaliste, puisque atteint pratiquement partout sur la planète, par la puissance des protocoles modernes de polychimiothérapie reposant sur l'utilisation de la rifampicine, ainsi que, par la suite, par une réduction « autoritaire » de la durée de traitement des sujets multibacillaires (passée de deux ans à un an en 1997). La définition purement clinique du caractère supposé multibacillaire ou paucibacillaire des patients, proposée également par cet organisme, doit être vue comme une approximation acceptable «sur le terrain», dans un contexte de dénuement et plus spécifiquement de peu de reproductibilité des techniques de mise en évidence des bacilles de la lèpre prévalant dans la plupart des zones d'endémie lépreuse.

Si ces raccourcis trouvent leur justification, et n'ont d'ailleurs pas compromis la sécurité des malades pour ce qui est du taux de rechute ou de résistance secondaire, le risque de démobilisation lié au succès de l'atteinte de l'objectif «d'élimination de la lèpre » ne peut, en revanche, plus être méconnu [15]. Le recentrage de la stratégie de l'OMS sur le diagnostic précoce des cas de lèpre (c'est-à-dire avant l'installation d'invalidités significatives) est effectif depuis 2007. Il apparaît désormais avéré qu'une mauvaise compréhension de l'objectif « d'élimination», parfois confondu avec une "éradication», dont on est bien loin [3], a contribué à une ambiance de désaffection générale vis-à-vis de la maladie, avec comme corollaires un tarissement des ressources de la recherche scientifique mais également des compétences pratiques dans ce domaine. 


\section{La prophylaxie est-elle « la » voie d'avenir?}

En réalité, les avancées récentes les plus excitantes concernent un domaine ancien mais qui a connu un regain d'actualité assez inattendu : celui de la prophylaxie. L'intérêt de l'administration d'une dose unique de rifampicine à des sujets sains contacts de cas de lèpre a été démontré à partir d'études très convaincantes menées sur des populations importantes et avec un recul dépassant désormais six ans [16]. En résumé, ce traitement apparaît à même de réduire l'incidence des nouveaux cas d'environ $57 \%$ parmi les contacts, ce d'autant plus qu'il s'agissait de contacts sans lien génétique avec le cas index. L'effet préventif d'une vaccination (ou d'une revaccination) par le BCG, vaccin connu de longue date pour conférer un certain degré de protection contre la lèpre, semble également significatif, et pourrait avoir un effet additif à la prise unique de rifampicine [17].

Ce type d'approche, même s'il est techniquement efficace, n'est toutefois pas sans poser d'importants problèmes opérationnels, notamment celui de la communication d'un diagnostic qui reste très stigmatisant à un entourage plus ou moins vaste. De plus, l'organisation de campagnes de distribution de traitements préventifs poserait d'évidents problèmes d'organisation sur le terrain, cela même qu'on a cherché à réduire jusqu'à présent... D'un autre côté, il pourrait s'agir d'un moyen efficace de résoudre le phénomène contrariant de stagnation d'incidence de la maladie constaté depuis une dizaine d'années, peut-être le seul. On attend avec intérêt une prise de position de l'OMS sur cette importante question.

\section{En conclusion : danger à l'horizon !}

Malgré des progrès certains dans la compréhension des mécanismes fondamentaux de la maladie, la démobilisation dans le domaine de la lutte contre la lèpre est une réalité. L'ignorance de ce problème représenterait un pari dont l'extraordinaire latence des événements jalonnant l'histoire naturelle de cette maladie suggère qu'il serait à haut risque. Au pire, et même si aucun élément ne le laisse supposer à l'heure actuelle, il ne faudrait pas qu'un réveil à moyen terme de l'endémie - «grand classique » de la médecine tropicale et des maladies négligées - ne survienne, d'autant plus qu'on ne disposerait guère alors de sentinelles capables de déceler précocement un tel événement. Depuis des siècles, on sait que la lèpre, maladie à l'indolence pernicieuse, se nourrit principalement de l'absence d'attention qu'on lui porte : faisons en sorte de ne pas oublier ce principe et de ne pas nous laisser endormir aussi bien par le rythme trompeur de la maladie que par un excès de triomphalisme qui ne serait absolument pas d'actualité [18]. $\diamond$

\section{SUMMARY}

Leprosy is definitely not a disease of the past

In this review we present a synthesis of the current knowledge of leprosy based on the epidemiological studies and the latest data obtained in basic research and the strategies undertaken to fight out this pathology. The significant progress in the genomic study of the leprosy bacillus, and in understanding the basic mechanisms governing the individual susceptibility to the disease, together with the stagnation in the incidence of the new cases observed for ten years, represent focus of interest. If the efficiency of the recommended treatments is actually confirmed by a low relapse rate, however the drying up of new cases, which could be expected by reducing the infectiousness of the treated cases, is nonetheless slow in coming. Recent studies support the effectiveness of prophylaxis based on a single dose of rifampicin and/or a BCG vaccination of patient contacts. This could allow an increased reduction of the incidence of the disease. However, no official position validates such strategies. Because of an ambiguity in the expression of the objectives for managing the disease, i.e. "elimination of a disease considered as a public health problem», which should not to be confused with « eradication of the disease », which, in fact, has no relevance today, demobilization in fighting against leprosy is to be feared. $\diamond$

\section{LIENS D'INTÉRêT}

L'auteur déclare n'avoir aucun lien d'intérêt concernant les données publiées dans cet article.

\section{RÉFÉRENCES}

1. De Carsalade GY, Mahé A. Lèpre. In : Mokni M, Dupin N, del Giudice P, eds. Dermatologie infectieuse. Paris : Elsevier-Masson, 2014 : 125-37.

2. Le point sur la situation mondiale de la lèpre, 2013 : réduction de la charge de morbidité. Wkly Epidemiol Rec 2014 ; 89 : 389-400.

3. Meima A, Smith WC, van Oortmarssen GJ, et al. The future incidence of leprosy: a scenario analysis. Bull World Health Org 2004 ; 82 : 373-80.

4. Cole ST, Eiglmeier K, Parkhill J, et al. Massive gene decay in the leprosy bacillus. Nature $2001 ; 409$ : 1007-11.

5. Cambau $\varepsilon$, Chauffour-Nevejans A, Tejmar-Kolar L, et al. Detection of antibiotic resistance in leprosy using GenoType LepraeDR, a novel readyto-use molecular test. PloS Negl Trop Dis 2012 ; 6 : el739.

6. Monot M, Honoré N, Garnier T, et al. On the origin of leprosy. Science 2005 ; $308: 1040-2$.

7. Monot M, Honoré N, Garnier T, et al. Comparative genomic end phylogeographic analysis of Mycobacterium leprae. Nat Genet 2009; 41 : 1282-9.

8. Truman RW, Pushpendra S, Sharma R, et al. Probable zoonotic leprosy in the Southern United States. N Engl J Med 2011 ; 364 : 1626-33.

9. Han XY, Jessurun J. Severe leprosy reactions due to Mycobacterium lepromatosis. Am J Med Sci 2013 ; 345 : 65-9.

10. Singh P, Benjak A, Schuenemann VJ, et al. Insight into the evolution and origin of leprosy bacilli from the genome sequence of Mycobacterium lepromatosis. Proc Natl Acad Sci USA 2015 ; 112 : 4459-64.

11. Ranque $B$, Alter $A$, Schurr $\varepsilon$, et al. La lèpre: un paradigme pour l'étude de la prédisposition génétique aux maladies infectieuses. Med Sci (Paris) 2008 ; $24: 491-7$.

12. Schollard DM, Adams LB, Gillis TP, et al. The continuing challenges of leprosy. Clin Microbiol Rev $2006 ; 19: 338-81$.

13. Faye 0 , N'Diaye HT, Keita S, et al. High prevalence of non-leprotic hypochromic patches among children in a rural area of Mali, West Africa. Lepr Rev 2005 ; 76 : 144-6. 


\section{RÉFÉRENCES}

14. Wagenaar I, Brandsma W, Post $\varepsilon$, et al. Two randomized controlled clinical trials to study the effectiveness of prednisolone treatment in preventing and restoring clinical nerve function loss in leprosy: the TENLEP study protocols. BMC Neurol $2012 ; 12$ : 159 .

15. Lockwood D, Shetty V, Penna GO. Hazards of setting targets to eliminate disease: lessons from the leprosy elimination campaign. BMJ $2014 ; 348:$ gl136.

16. Moet FJ, Pahan D, Oskam L, Richardus JH. Effectiveness of single dose rifampicin in preventing leprosy in close contacts of patients with newly diagnosed leprosy: cluster randomised controlled trial. BMJ 2008 ; $336: 761-4$.

17. Richardus RA, Alam K, Pahan D, et al. The combined effect of chemoprophylaxis with single dose rifampicin and immunoprophylaxis with BCG to prevent leprosy in contacts of newly diagnosed cases: a cluster randomized controlled trial (MALTALEP study). BMC Infect Dis $2013 ; 13: 456$.
18. Bulletin de l'Association des Léprologues de Langue Française (BALLF) disponible en ligne sur http://www.leprosy-information.org/resource/ bulletin-de-lallf-revue-francophone-d-information-sur-la-lepre-etI-ulcere-de-buruli

19. Corti 0 . Une ubiquitine ligase polyvalente : garante de la qualité mitochondriale et de l'immunité antibactérienne? Med Sci (Paris) 2014 $30: 350-2$.

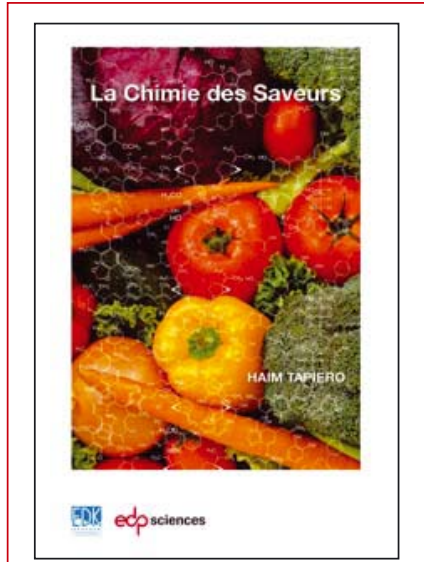

a cuisine est une science. II existe une relation étroite entre élaborer une recette et entreprendre une recherche scientifique. Quelle que soit l'origine d'une recette, d'un livre ou inventée, il faudra faire le choix des ingrédients, les mélanger et les cuire de manière appropriée afin de ne pas altérer les substances actives qui composent les ingrédients.

Une fois la cuisson terminée, il faudra analyser le goût et si nécessaire prévoir son amélioration. Améliorer une recette nécessite de connaître le ou les processus qui interviennent dans le développement des arômes, des saveurs et de la texture. Cette approche est similaire à celle développée par le scientifique.

La relation entre l'élaboration des recettes, les substances nutritives qui composent les ingrédients et la santé de l'homme est issue de plusieurs disciplines de la recherche fondamentale et clinique. Au cours des dernières années, de nombreux travaux scientifiques ont été publiés sur le rôle de la nutrition et la réduction des risques dans les pathologies comme les maladies cardio-vasculaires ou les cancers.

Le but principal de cet ouvrage a été d'identifier la structure chimique des composants actifs des ingrédients utilisés en cuisine (légumes, herbes aromatiques, épices) et qui entrent dans la préparation des recettes pour « végétariens » et « omnivores ».

ISBN : 978-2-7598-1137-3 180 pages

À retourner à EDK, 109, avenue Aristide Briand, 92541 Montrouge Cedex - Tél. : 0141177405 - Fax : 0149850345 - E-mail : edk@edk.fr NOM : Prénom :

Adresse :

Code postal :

Ville :

Pays :

Fonction :

Je souhaite recevoir l'ouvrage La chimie des Saveurs : $20 €+3 €$ de port $=\mathbf{2 3} €$ TTC

en ................ exemplaire, soit un total de

$\ldots$

$\square$ Par chèque, à l'ordre de $\mathbf{E} \mathbf{D} \mathbf{K}$

$\square$ Par carte bancaire : $\quad \square$ Visa $\square$ Eurocard/Mastercard

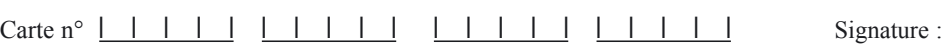

Date d'expiration :

$1 \quad 1 \quad 1 \quad 1$

$\mathrm{N}^{\circ}$ de contrôle au dos de la carte :

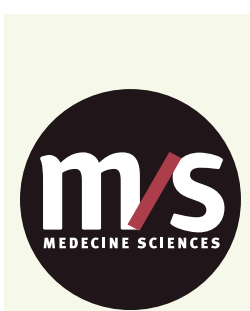

Tarifs d'abonnement $\mathrm{m} / \mathrm{s}-2015$

Abonnez-vous

à médecine/sciences
$>$ Grâce à $m / s$, vivez en direct les progrès des sciences biologiques et médicales

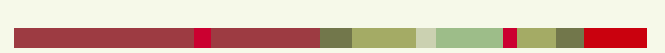

\section{Bulletin d'abonnement page 734 dans ce numéro de $\mathrm{m} / \mathrm{s}$}

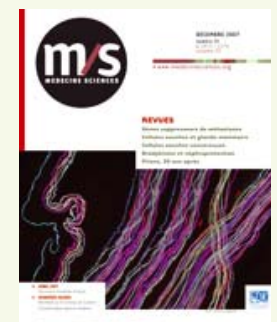

